\title{
Lattice Polygons in the Plane and the Number 12
}

\author{
JOHN M. BURNS AND DAVID O'KEEFFE
}

\section{INTRODUCTION}

A convex polygon $\mathcal{P}$ in $\mathbb{R}^{2}$ all of whose vertices have integer coordinates is called a convex lattice polygon. If the polygon has $n$ lattice points on its boundary, represented by the vectors $p_{1}, \ldots, p_{n}$ (in anticlockwise order), then we say that the length $l(\mathcal{P})$ is $n$. The dual (convex) lattice polygon $\mathcal{P}^{\vee}$ is by definition the convex hull of the difference vectors $q_{i}=p_{i+1}-p_{i}$, where indices throughout the note are considered modulo $n$. In this note we will give a simple proof of the fact that if $\mathcal{P}$ be a convex lattice polygon in $\mathbb{R}^{2}$ whose only interior lattice point is the origin, then $l(\mathcal{P})+l\left(\mathcal{P}^{\vee}\right)=12$. This result has its origin in a correspondence between convex lattice polygons and certain toric varieties, and has several ingenious proofs (see [1] and [2]). These proofs use either Noether's formula or modular forms to explain the occurrence of the number 12. Our proof observes that if $l(\mathcal{P})$ increases by one then $l\left(\mathcal{P}^{\vee}\right)$ decreases by one, so that their sum is constant. The number 12 then appears as 3 (the smallest possible length) plus 9 (the largest possible length.) Our proof (which grew out of the second authors project, supervised by the first author) also supports the suggestion in [2] that the number 3 can be viewed as a discrete analogue of $\pi$ in this context.

\section{The Theorem}

Theorem 2.1. Let $\mathcal{P}$ be a convex lattice polygon in $\mathbb{R}^{2}$ whose only interior lattice point is the origin, and let $\mathcal{P} \vee$ be its dual. Then $l(\mathcal{P})+l\left(\mathcal{P}^{\vee}\right)=12$.

It is not hard to see that up to the action of $G L(2, \mathbb{Z})$ there are 16 such polygons but we will not need this fact. We will however use the following result of P. Scott [3], the proof of which relies only on elementary geometry. 
Lemma 2.1. Let $\mathcal{P}$ be a convex lattice polygon in the plane with $l(\mathcal{P})$ boundary lattice points and $c \geq 1$ interior points then $l(\mathcal{P}) \leq 2 c+7$.

We will also use picks formula, which in our context states:

Lemma 2.2. The area of a lattice polygon $\mathcal{P}$ is given by the formula $A(\mathcal{P})=c+l(\mathcal{P}) / 2-1$.

In our case $c=1$, so that $l(\mathcal{P}) \leq 9$ and $A(\mathcal{P})=l(\mathcal{P}) / 2$. Since we will think of the plane as the complex plane $\mathbb{C}$, we will label the vectors $p_{i}$ and $q_{i}$ as $z_{i}$ and $w_{i}$ respectively and we let $\gamma_{i}$ denote the straight line path (parameterised on $[0,1]$ ) joining $z_{i}$ to $z_{i+1}$. We will fix $z_{1}=1$ throughout unless we state otherwise. We recall that for a piecewise smooth curve $\gamma$ in $\mathbb{C}$ we have $\int_{\gamma} \bar{z} d z=2 i A$ where $A$ denotes the area enclosed by $\gamma$. In particular a convex lattice polygon with vertices $z_{1}, \ldots, z_{n}$ has area

$$
\begin{aligned}
-\frac{i}{2} \sum_{i=1}^{n} \int_{0}^{1} & \left(\overline{t z_{i+1}+(1-t) z_{i}}\right)\left(z_{i+1}-z_{i}\right) d t \\
& =-\frac{i}{4} \sum_{i=1}^{n}\left[\left|z_{i+1}\right|^{2}-\left|z_{i}\right|^{2}+\bar{z}_{i} z_{i+1}-z_{i} \bar{z}_{i+1}\right] \\
& =-\frac{i}{4} \sum_{i=1}^{n}\left[\bar{z}_{i} z_{i+1}-z_{i} \bar{z}_{i+1}\right] .
\end{aligned}
$$

It will be convenient to introduce the notation $A_{i j}=-\frac{i}{4}\left(\bar{z}_{i} z_{j}-z_{i} \bar{z}_{j}\right)$, namely the signed area of the oriented triangle with vertices $o, z_{i}, z_{j}$. Similarly, we let $A_{i j}^{\vee}=-\frac{i}{4}\left(\bar{w}_{i} w_{j}-w_{i} \bar{w}_{j}\right)$, so that

$$
A_{i i+1}^{\vee}=-\frac{i}{4}\left(\bar{z}_{i} z_{i+1}-z_{i} \bar{z}_{i+1}+\bar{z}_{i+1} z_{i+}-z_{i+1} \bar{z}_{i+2}+\bar{z}_{i+2} z_{i}-z_{i} \bar{z}_{i+2}\right) \text {. }
$$

In summary $A_{i i+1}^{\vee}=A_{i i+2}+A_{i+1 i+2}+A_{i+2 i}$, so that $l\left(\mathcal{P}^{\vee}\right)=2 l(\mathcal{P})-$ $\sum_{i=1}^{n} A_{i i+2}$. This immediately yields that when $l(\mathcal{P})=n=3$ we have $l\left(\mathcal{P}^{\vee}\right)=A_{12}^{\vee}+A_{23}^{\vee}+A_{31}^{\vee}=\left(A_{12}+A_{23}+A_{31}\right)+\left(A_{23}+A_{31}+A_{12}\right)+$ $\left(A_{31}+A_{12}+A_{23}\right)=3\left(A_{31}+A_{12}+A_{23}\right)=3 \times 3=9$ (since $A_{i i+1}=1$ for all $i$ ). If $l(\mathcal{P})=n=4, \sum_{i=1}^{n} A_{i i+2}=A_{13}+A_{24}+A_{31}+A_{42}=0$ (since $A_{i j}=-A_{j i} \forall i, j$ ) and we have $l\left(\mathcal{P}^{\vee}\right)=2 l(\mathcal{P})=8$. In future we will denote the sum $\sum_{i=1}^{n} A_{i i+2}$ by $d(\mathcal{P})$, and show that it increases by 3 when $l(\mathcal{P})$ increases by 1 , thus keeping $l(\mathcal{P})+l\left(\mathcal{P}^{\vee}\right)$ constant.

Proof. The case $l(\mathcal{P})=n=5$ is the crucial case as all others essentially follow from this one. Here since $A(\mathcal{P})=5 / 2$ and no edge 
contains more than 3 vertices, we may centre $\mathcal{P}$ at the origin with its vertices on the unit square. Since one of the 4 lines $x=0, y=0$, $y= \pm x$ must contain 2 of the 5 vertices of $\mathcal{P}$, we will assume that it is the $x$-axis. We may also assume that $2\left(z_{2}\right.$ and $\left.z_{3}\right)$ of the remaining 3 vertices lie above the $x$-axis and that the remaining vertex $z_{5}$ lies below it. It is immediate that $A_{41}=0\left(z_{4}, o\right.$, and $z_{1}$ being collinear). We first consider the case where $\mathcal{P}$ has an edge of length 2 (i.e., containing 3 vertices). This edge consists either of the points $\left\{z_{5}, z_{1}, z_{2}\right\}$ or $\left\{z_{3}, z_{4}, z_{5}\right\}$, so that either $A_{52}=2$ or $A_{35}=2$. In the former case $A_{35}=-1$, and in the latter $A_{52}=-1$. In both cases the remaining $A_{i i+2}$ are equal to 1 , so that $\sum_{i=1}^{n} A_{i i+2}=3$ and we are done. When $\mathcal{P}$ has no edge of length 2 , we must have an additional $A_{i i+2}=0$ on account of the fact that $z_{5}$ doesn't lie on a vertical edge of length 2 . This forces the remaining $A_{i i+2}$ to be 1 , and again $\sum_{i=1}^{n} A_{i i+2}=3$. When $l(\mathcal{P})=6$, we can (after relabelling if necessary) delete the vertex $z_{6}$ from $\mathcal{P}$ to obtain a convex lattice polygon $\mathcal{P}^{\prime}$ containing the origin, and $d(\mathcal{P})-d\left(\mathcal{P}^{\prime}\right)=$ $A_{13}+A_{35}+A_{51}+A_{24}+A_{46}+A_{62}-\left(A_{13}+A_{35}+A_{52}+A_{24}+A_{41}\right)=$ $A_{51}+A_{46}+A_{62}+A_{25}+A_{14}=3$. The last equality follows from the observation that $A_{51}+A_{46}+A_{62}+A_{25}+A_{14}=d\left(\mathcal{P}^{\prime \prime}\right)$ where $\mathcal{P}^{\prime \prime}$ is either the convex lattice pentagon $\left\{z_{1}, z_{2}, z_{4}, z_{5}, z_{6}\right\}$ containing the origin, so that $d\left(\mathcal{P}^{\prime \prime}\right)=3$ by above, or else the convex lattice hexagon (the above pentagon with the origin adjoined) with no interior lattice point, where the computation is trivial. We now have $l\left(\mathcal{P}^{\vee}\right)=2 l(\mathcal{P})-\left(d\left(\mathcal{P}^{\prime}\right)+3\right)=12-6=6$. It is intriguing that the cases $l(\mathcal{P})=7,8,9$ are identical to that of $l(\mathcal{P})=6$. In each case, just as above $d(\mathcal{P})-d\left(\mathcal{P}^{\prime}\right)=d\left(\mathcal{P}^{\prime \prime}\right)$ where $\mathcal{P}^{\prime \prime}$ is either a convex lattice pentagon containing the origin, or the convex lattice hexagon with no interior lattice point, so that $d(\mathcal{P})-d\left(\mathcal{P}^{\prime}\right)=3$.

Remark. Finally we point out some connections with [2]. There it is shown that the vectors $p_{i}$ and $p_{i+1}$ form a basis for the lattice $\mathbb{Z}^{2}$ with the same orientation as the standard basis $\{(1,0),(0,1)\}$, and that there are matrices $M_{i}=\left(\begin{array}{rr}0 & 1 \\ -1 & d_{i}\end{array}\right)$ in $S L(2, \mathbb{Z})$ such that $M_{i}\left(\begin{array}{r}p_{i-1} \\ p_{i}\end{array}\right)=\left(\begin{array}{r}p_{i} \\ p_{i+1}\end{array}\right)$, where the $2 \times 2$ matrices $\left(\begin{array}{r}p_{i} \\ p_{i+1}\end{array}\right)$ have the row vectors $p_{i}$ and $p_{i+1}$ as their rows. In addition we have that

$$
\left(\begin{array}{rr}
0 & 1 \\
-1 & d_{n}
\end{array}\right)\left(\begin{array}{rr}
0 & 1 \\
-1 & d_{n-1}
\end{array}\right) \cdots\left(\begin{array}{rr}
0 & 1 \\
-1 & d_{1}
\end{array}\right)=I
$$


the identity matrix. It is a simple matter to see that in fact $d_{i}=$ $A_{i i+2}$ and that the above equations therefore contain some of our observations above. When $n=3$ they imply that $A_{13}=A_{32}=$ $A_{21}=-1$. When $n=4$ they imply that $A_{i i+2}=-A_{i+2 i} \forall i$. When $n=5$ they imply that the multi-set $\left\{A_{13}, A_{35}, A_{52}, A_{24}, A_{41}\right\}$ is either the multi-set $\{-1,2,1,0,1\}$ or else $\{0,1,1,0,1\}$. In either case $d(\mathcal{P})=\sum_{i=1}^{n} A_{i i+2}=3$. We also observe that our proof supports the suggestion in [2] that 3 can be viewed as a discrete analogue of $\pi$. Clearly $d(\mathcal{P})$ has an interpretation as the sum of the "discrete exterior angles" of $\mathcal{P}$ (as defined in [2].) We have shown that increasing $l(\mathcal{P})$ by one increases this sum by 3 , whereas the sum of the exterior angles increases by $\pi$ for a general polygon.

\section{REFERENCES}

[1] W. Fulton, Introduction to toric varieties, Annals of Mathematics Studies, No. 82, Princeton University Press 1993.

[2] B. Poonen and F. Rodriguez-Villegas, Lattice polygons and the number 12, Amer. Math. Monthly 107 (2000), 238-250.

[3] P. Scott, On convex lattice polygons, Bull. Austral. Math. Soc. 15 (1976), 395-399.

John M. Burns and David O'Keeffe,

Department of Mathematics

National University of Ireland,

Galway, Ireland

john.burns@nuigalway.ie

Received on 25 October 2005. 